\title{
THE ORIGIN OF FORBIDDEN LINE EMISSION FROM YOUNG STELLAR OBJECTS
}

\author{
Ana I. Gomez de Castro ${ }^{1,2,3}$ AND Ralph E. Pudritz ${ }^{1,4}$ \\ Received 1991 August 15 ; accepted 1992 November 2
}

\begin{abstract}
We present a model for the origin of blueshifted, optical forbidden line emission and jets in young stellar objects based on generic properties of hydromagnetic disk winds. Magnetic stresses recollimate hydromagnetic disk winds to magnetic focal regions under very general conditions. We demonstrate that conditions in MHD shocks at these points account for the observed emission. We find that for fiducial accretion rates of $10^{-7} M_{\odot}$ $\mathrm{yr}^{-1}$ and magnetic fields at the inner edge of the disk $\left(\simeq 10^{12}\right) \mathrm{cm}$, gas accelerated from the innermost parts of a Keplerian accretion disk focuses into regions $\geq 0.4 \mathrm{AU}$ in radius that lie $\simeq 16 \mathrm{AU}$ above and below the disk. The shocked gas density ranges from $\simeq 10^{4}$ to $10^{8} \mathrm{~cm}^{-3}$ where the latter occurs in the innermost part of the flow and shock. Shocked gas speeds range up to $250 \mathrm{~km} \mathrm{~s}^{-1}$ (going from the outer part of the shock at $\simeq 2 \mathrm{AU}$, to the innermost region) under these conditions. The magnetic field is moderately amplified in the shock and diverges from the flow axis in the postshock flow. It is this feature of MHD shock that produces an expanding cone of shocked gas. The opening angle of the postshock gas with respect to the flow axis is $40^{\circ}$, and this accounts for the double-peaked character of the line profiles. Our model also predicts that the slower velocity component is associated with shocked gas of lower density than that associated with the higher velocity component. We show that the wind is largely neutral with an electron fraction of $10^{-1}$. The wind remains largely neutral in adiabatic MHD shocks because much of the preshock kinetic energy goes into an increased postshock magnetic field. Substantial fractions of the flow energy can be liberated in these shocks.
\end{abstract}

Subject headings: accretion, accretion disks - shock waves - stars: emission-line, Be — stars: mass loss stars: pre-main-sequence

\section{INTRODUCTION}

Bipolar molecular outflows appear to be the earliest signature of star formation. It has become clear that most, if not all, low-mass stars undergo this outflow phase and that the flow lifetimes are typically of order $2 \times 10^{5}$ yr (Parker, Padman, \& Scott 1991). Manifestations of outflow are observed on a wide range of spatial scales down to those $\simeq 100 \mathrm{AU}$ characterizing the optical forbidden line emission ([O I $],[\mathrm{S} \mathrm{II}],[\mathrm{N}$ II $]$ ) region (FLER) (Jankovics, Appenzeller, \& Krautter 1983; Edwards et al. 1987). Observations by Edwards et al. (1987) and Cabrit et al. (1990) have shown that forbidden line emission is strongly correlated with infrared excess which they interpret as a correlation with the presence of circumstellar accretion disks. Another observational clue that suggests that bipolar outflows originate from accretion disks and not their central stars comes from the detailed studies of line profiles of $\mathrm{Li} \mathrm{I}, \mathrm{Fe} \mathrm{I,} \mathrm{Fe} \mathrm{II,} \mathrm{and}$ Ti I line profiles in FU Ori systems (Calvet, Hartmann, \& Kenyon 1993). Wind originating from the innermost part of a Keplerian disk rotates much more quickly than that from the more distant regions. The resulting differential behavior of the line profiles (strong lines from the innermost parts of the disk are more asymmetric than weaker line emission from the outer parts) predicted by disk wind theory is successful in accounting for these trends. Stellar winds do not readily account for such observations. The idea that bipolar outflows and molecular disks are dynamically coupled was proposed in early theoretical models of hydromagnetic disk winds (Pudritz \& Norman 1983; Pudritz 1985).

\footnotetext{
${ }^{1}$ Department of Physics and Astronomy, McMaster University, Hamilton, Ontario, Canada L8S 4MI.

2 NSERC International Postdoctoral Fellow.

${ }^{3}$ Present Address, IUE Observatory-VILSPA, P.O. Box 50727, 28080, Madrid, Spain.

${ }^{4}$ CITA, University of Toronto, Toronto.
}

Theoretical hydrodynamic models for the forbidden line emission from young stellar objects (YSOs) emphasize oblique shocks because low-excitation lines are observed in highvelocity postshock gas. Hartmann \& Raymond (1988) were able to find reasonable fits to the observed line profiles by assuming that a stellar wind shocks with a surrounding, flaring disk. In such a theory, one would anticipate that the forbidden line emission would lie in the plane of the disk if one could resolve it. Given the increasingly direct observational support for the existence of disk winds, do FLERs also have an explanation in the context of disk wind theory?

In this paper we show that line formation in recollimating hydromagnetic disk winds is a direct consequence of disk wind theory. The central reason why such behavior is expected is because of the generic ability of magnetic tension from the toroidal magnetic field in hydromagnetic disk winds to collimate the flow and under the right conditions to recollimate it toward the axis (Blandford \& Payne 1982, hereafter BP; Pelletier \& Pudritz 1992, hereafter PP). The transverse recollimation of flow will produce oblique shocks (e.g., Achterberg, Blandford, \& Goldreich 1983), and we show that forbidden line emission and optical jets naturally arise at these focal points. The line emission should be observed to be produced from small regions symmetrically placed along the flow axis, above and below the accretion disk. Since the source of the outflow is the accretion disk we emphasize that the evolution of the flow should be intrinsically coupled to the evolution of the disk.

We summarize the observed characteristics of $T$ Tauri emission lines by the direct approach of using the infrared colorcolor diagram, $(H-K, K-L)(\S 2)$. We state the analytical results of disk wind theory of PP that we require for this work in $\S 3$. We then use simple MHD shock jump conditions at the magnetic focal points to calculate the typical conditions of the shocked flow there $(\S 4)$ and show that the emission from the 
shocked gas readily accounts for the forbidden line emission $(\S 5)$. We briefly discuss links between forbidden line production, disk winds, and the observed properties of disks (§ 6). A preliminary account of this work appears in Pudritz, Pelletier, \& Gomez de Castro (1991).

\section{WIND DIAGNOSTICS: THE INFRARED COLOR DIAGRAM}

We use a $H-K, K-L$ diagram to plot the relationship between observed wind indicators and infrared excess in the T Tauri stars (TTSs). This is essentially an HR diagram (luminosity versus effective temperature) where the characteristics of the dust envelope surrounding the post-mainsequence (PMS) stars is given the largest weight. We use this diagram because the large differences among the TTSs in the various mass-loss and accretion indicators (such as emissionline strength, veiling, UV excess, etc.) show up very well. Since the infrared spectra of YSOs evolve systematically (e.g., Lada 1991), an obvious approach is to look for correlations of physical properties in an infrared color-color diagram. The choice of the $H-K, K-L$ colors is a compromise between selecting a stellar contribtion that is as small as possible, and having a large enough data set available.

We have selected for our data set all TTSs in Taurus since they comprise the best-studied sample of low-mass protostars associated with a single star-forming region. We have not corrected the colors for interstellar extinction since the region is nearby, $d \simeq 140 \mathrm{pc}$, and the interstellar extinction in this direction is very small. The TTSs have been divided into two categories based upon the presence of [O I] $\lambda 6300$ emission and of photospheric features by Cohen, Emerson, \& Beichman (1989). A third obvious group of TTSs, are the so-called Weak TTS (WTTSs). These have weak $\mathrm{H} \alpha$ emission and strong chromospheric activity. Summarizing the data:

1. Strong [O $\mathrm{I}]$ emission and continuum stars,

2. Faint $\left[\mathrm{O}_{\mathrm{I}}\right]$ emission and normal TTSs, and

3. LkCa II stars and X-ray sources.

In Figure 1 we plot all members of the above categories that have published infrared colors (TTSs: from Rydgren, Strom, \& Strom 1976, Cohen \& Kuhi 1979, and Elias 1978; LkCa II stars: Herbig, Vrba, \& Rydgren 1986; and X-ray sources: Walter et al. 1988). Figure 1 has not been corrected for extinction since this is very small. The values of $A_{v}$ in the stars located in regions $\mathrm{B}$ and $\mathrm{C}$ are smaller than $1 \mathrm{mag}$ (Strom et al. 1990; Cabrit et al. 1990), which implies excesses in $H-K$ and $K-L$ of $\simeq 0.06 \mathrm{mag}$. This accounts for the intermingling of sources at the boundaries in Figure 1.

A striking sequence is observed in this figure. Sources marked by filled circles belong to type I and are concentrated in the upper right corner of the diagram (region A) and are therefore highly embedded. Sources marked with open circles belong to type II, and these appear in the center of the diagram (region B) with little overlap with type I sources. Finally, type III sources (denoted by asterisks and crosses) are found preferentially in the lower left-hand corner (region $\mathrm{C}$ ) and do not overlap with type II sources. Thus, as one moves from type I to III, both $H-K$ and $K-L$ colors become bluer, that is, there is an extremely good correlation with infrared excess. A second striking aspect of the diagram is that all TTSs in the Taurus cloud that have associated $\mathrm{HH}$ objects, jets, or forbidden line emission regions $\geq 2$ " ( $\geq 320$ AU at distance of Taurus) are located in region A of our diagram and are designated by filled squares (see Table 2 in Appenzeller \& Mundt 1989).

In Figure 2 we quantify this trend by plotting the infrared

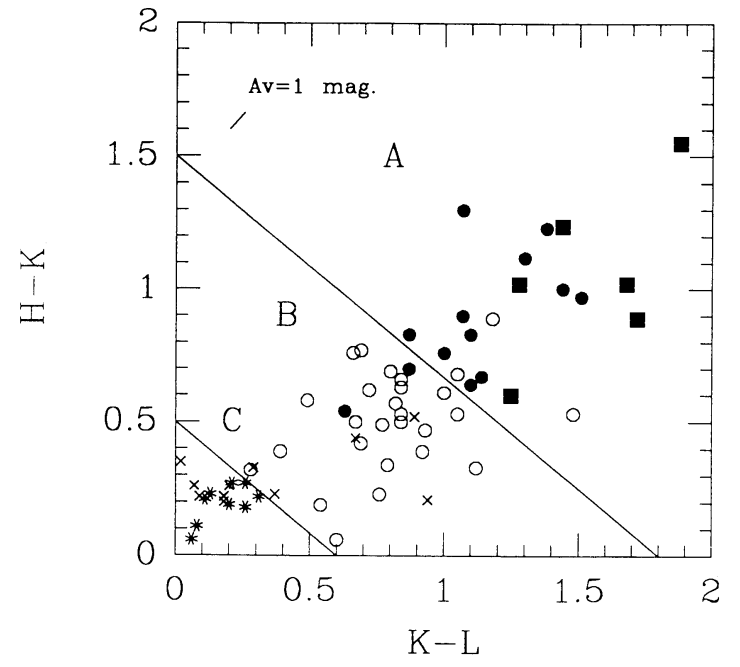

FIG. 1. $-H-K, K-L$ diagram of the PMS sources in Taurus. The diagram is divided in three regions depending on the color excesses of the sources. Filled circles represent strong emission line (type I) sources. Filled squares are sources with well-studied optical jets. Open circles are weak emission-line sources (type II). Asterisks and crosses are the $\mathrm{X}$-ray and $\mathrm{LkCa}$ II sources (type III), respectively.

colors of stars with measured [O I] and $\mathrm{H} \alpha$ equivalent widths, respectively (data from Cohen \& Kuhi 1979; Cabrit et al. 1990; Strom et al. 1990). The filled circles in Figure $2 a$ represent stars with equivalent widths $W\left(\left[\mathrm{O}_{\mathrm{I}}\right]\right)>1.0 \AA$ while the open circles

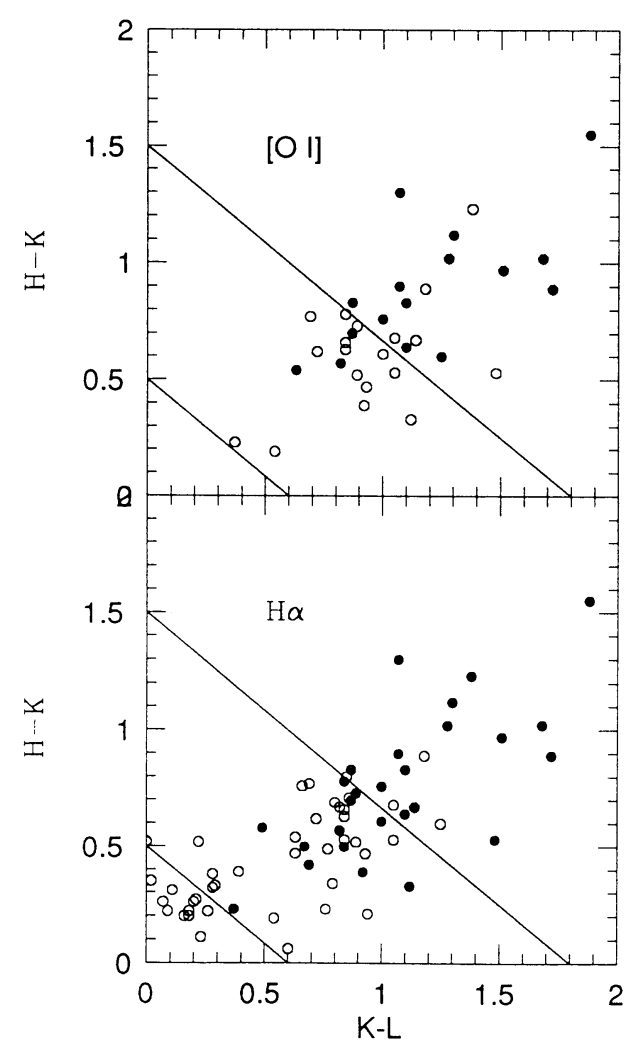

FIG. 2.-(a) $H-K, K-L$ diagram of those PMS sources in Taurus with measured $\left[\mathrm{O}_{\mathrm{I}}\right]$ emission. Filled and open circles represent stars with $W([\mathrm{O} \mathrm{I}])>1.0 \AA$ and $W\left(\left[\mathrm{O}_{\mathrm{I}}\right]\right) \leq 1.0 \AA$, respectively; $(b) H-K, K-L$ diagram of those PMS sources in Taurus with measured $\mathrm{H} \alpha$ emission. Filled and open circles represent stars with $W(\mathrm{H} \alpha)>50 \AA$ and $W(H \alpha) \leq 50 \AA$, respectively. 
have $W\left(\left[\mathrm{O}_{\mathrm{I}}\right]\right)$ less than this value. In Figure $2 b$ the filled circles represent all objects with $W(\mathrm{H} \alpha)>50 \AA$ while the open circles have $W(\mathrm{H} \alpha)$ less than this value. The stars in region $\mathrm{C}$ have $W(\mathrm{H} \alpha)<5.0 \AA$.

If outflows are hydromagnetic stellar winds, then there should be a strong correlation between wind diagnostics and stellar rotation. In order to check this, we compiled the data of Hartmann et al. (1986) and Hartmann \& Stauffer (1989) who measured the rotational velocities for a large number of PMS stars in Taurus. We divide these data into three groups with $v \sin i<15 ; 15 \leq v \sin i \leq 20$, and $v \sin i>20 \mathrm{~km} \mathrm{~s}^{-1}$ and plot them in the color-color diagram (Fig. 3). It is clear that there is no correlation of stellar $v \sin i$ with infrared color. Thus for any color $K-L$ in our diagram, we can find both quickly and slowly rotating stars.

It is a difficult task to measure the rotation speed of the more deeply embedded objects. The most exotic sources, such as those that have fully developed optical jets, are also the most deeply embedded systems as is seen in Figure 1. Nonetheless, good measurements exist for the rotation of the underlying stars even for some of these systems. Thus, two of the TTSs with associated optical jets in Figure 1, T Tau and DG Tau, have measured $v \sin i$ values of $20.1 \pm 1.5$ and $21.7 \pm 6.3 \mathrm{~km}$ $\mathrm{s}^{-1}$, respectively. On the other hand, a star such as FP Tau that is on the boundary of regions $B$ and $C$ in Figure 1 has a high measured $v \sin i$ of $26.6 \pm 1.6 \mathrm{~km} \mathrm{~s}^{-1}$.

It is clear from these two figures that while the rotation speeds of the underlying TTSs are completely uncorrelated with near-infrared colors, emission-line properties are very well correlated. It is therefore highly unlikely that stellar rotation has much to do with the production of the forbidden line regions in TTSs. The same conclusions pertain for boundary layer winds, since for these to be effective, rotation rates must be near "breakup." Since line emission is produced in shocks associated with outflow, a more natural explanation is that wind from surrounding disks accounts for the correlations found in Figure 1. The task is to delineate a general reason for the presence of shocks in such winds and why these might correlate with near-infrared color. We first examine the generic

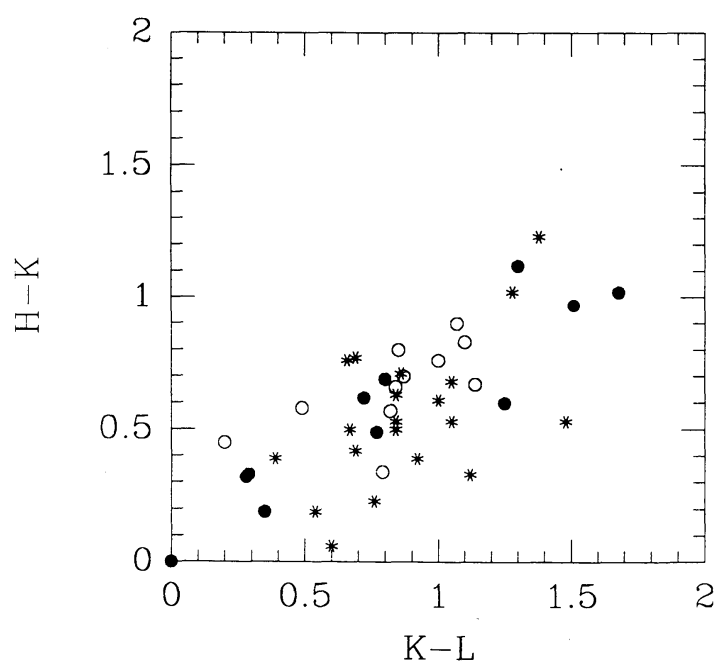

Fig. 3. $-H-K, K-L$ diagram of those PMS sources in Taurus with measured $v \sin i$. Asterisks denote $v \sin i<15 \mathrm{~km} \mathrm{~s}^{-1}$, open circles $15 \leq$ $v \sin i \leq 20 \mathrm{~km} \mathrm{~s}^{-1}$, and filled circles $v \sin i>20 \mathrm{~km} \mathrm{~s}^{-1}$. features of hydromagnetic disk winds that are important for understanding the origin of shocks.

\section{HYDROMAGNETIC DISK WINDS}

It is now well established that accretion disks threaded by open magnetic field lines of sufficient strength can drive coronal gas from the disk to form high-speed jets and bipolar outflows (BP; Pudritz 1985; Konigl 1989; PP). The outflows are driven by the component of centrifugal force upon a rotating gas particle that is directed parallel to the magnetic field line it finds itself upon.

A second fundamental attribute of the hydromagnetic flows from disks is that the wind mass-loss rate must necessarily be related to the accretion rate through the underlying disk because it is the wind that carries off the disk material's angular momentum. Since the magnetic fields that thread a disk force the corotation of accelerating particles out to large distances $r_{\mathrm{A}}$, the wind mass-loss rate can be tiny compared to the disk accretion flow that it helps to sustain.

The outflows as we have seen are necessarily highly supersonic, but they are also highly super-Alfvénic. Rotating hydromagnetic outflows develop strong toroidal magnetic fields far from the base of the wind. It is accordingly convenient to divide the total magnetic field of the wind into toroidal and poloidal components, $\boldsymbol{B}=\boldsymbol{B}_{\phi}+\boldsymbol{B}_{\boldsymbol{p}}$. The rotational velocity of the flow is only a small fraction of the poloidal (i.e., bipolar) component of the outflow.

A third important important feature of rotating hydromagnetic flows is that they self-collimate. The ever-increasing toroidal field in the outflow $\left(B_{\phi}\right)$ implies that the pinching $\boldsymbol{J}_{\boldsymbol{p}} \times \boldsymbol{B}_{\phi}$ force must become significant as well. Detailed calculations (BP; PP; Heyvaerts \& Norman 1989) show that this force overcomes the magnetic and gas pressure of the jet, as well as the centrifugal stress of the rotating gas and collimates the flow far from the disk.

For a gas parcel that begins its outflow on a field line that threads the disk at the cylindrical radius $r_{0}$ in the corona near the disk surface, then the Alfven point on that field line occurs at a cylindrical distance $r_{\mathrm{A}}\left(r_{0}\right)$ from the axis. The significance of the Alfvén surface is that the flow reaches the Alfvén speed in the polidal magnetic field $V_{\mathrm{A}, p}$ at this point. This Alfvén speed is one of three characteristic signal speeds in a magnetized gas. The Alfven surface has the added significance that beyond it, the magnetic field can no longer keep an accelerating gas parcel in strict corotation with the underlying source so that further acceleration largely ceases.

It will prove convenient in what follows to define a magnetic Mach number of the hydromagnetic flow as the ratio of the poloidal speed of the flow to the total Alfvén speed,

$$
n \equiv \frac{v_{p}}{V_{\mathrm{A}}}
$$

where $v_{p}$ is the poloidal flow velocity and $V_{\mathrm{A}} \equiv$ $\left(B_{p}^{2}+B_{\phi}^{2}\right)^{1 / 2} /(4 \pi \rho)^{1 / 2}$ the Alfvén speed in the total magnetic field of the flow (the Mach number $n$ is often referred to as the "fast magnetosonic" [FM] number of the flow). For a cold flow, the fast magnetosonic wave is the last signal that can communicate back with the source. Beyond the surface where $n=1$ (known as the FM surface), the flow moves faster than the propagation speed of any hydromagnetic wave. It is well known that it is in this regime that the field becomes strongly toroidal, and it begins to collimate. 
The bulk of this section briefly summarizes the basics of hydromagnetic disk wind theory required for our analysis; the reader may consult $\mathrm{PP}$ for the details.

\subsection{Flow Energetics and Collimation}

In all that follows the flow is assumed to be axisymmetric, and hence cylindrical coordinates are used throughout. The magnetic field lines and the streamlines of the flow are contained in surfaces of constant magnetic flux $a=$ const. The poloidal component of the total field (everything that isn't toroidal) is derived from this scalar potential. A number of physical quantities are conserved for the component of the flow that is parallel to surfaces of constant flux such as the mass, angular momentum per unit mass $l(a)$, and total energy per unit mass $E(a)$. The latter is just Bernoulli's theorem for flow along streamlines. A simple consequence of the Bernoulli equation is that the terminal speed of the wind along any flux surface is

$$
v_{p, \infty} \simeq 2^{1 / 2} \Omega_{K} r_{\mathrm{A}}
$$

A complete theory of hydromagnetic flow also requires force balance in directions that are normal to any given magnetic surface from point to point in the flow. This condition generates a differential equation for the potential $a$, which is known as the Grad-Shafranov (G-S) equation. An important function $\lambda(a)$ appears in the G-S equation which governs the overall geoemetry of the outflow and which is nearly constant throughout the flow. Analytic solutions for flows can be found when $\lambda=$ const (PP).

\subsection{Two-dimensional Disk Wind: Basic Parameters}

Hydromagnetic winds originate near the surface of Keplerian (sufficiently close to the central star) accretion disks. In addition to the usual requirements of force balance (G-S) and energy conservation (Bernoulli) within the wind, the boundary condition is imposed that the wind carry off the disk angular momentum from each disk radius so that a steady mass accretion rate $\dot{M}_{a}$ is maintained within the disk.

In all generality BP and PP find that axisymmetric disk winds are governed by two parameters $\epsilon$ and $\lambda$ which measure the strength of the magnetic field threading the disk through some fiducial inner radius of the disk, $r_{i}$, and the angular momentum of the flow, respectively. For hydromagnetic winds from accretion disks around YSOs, the former is

$$
\epsilon_{i} \equiv \frac{\dot{M}_{a} \Omega_{i}}{3 r_{i} B_{i}^{2}}=0.24 \frac{\dot{m}_{a} m_{*}^{1 / 2}}{b_{i}^{2}\left(r_{i} / 10^{12} \mathrm{~cm}\right)^{5 / 2}},
$$

where PP assumed a fiducial inner disk radius of $r_{i}$ of $10^{12} \mathrm{~cm}$ around a star of mass $m$ (measured in units of a solar mass $\left.M_{\odot}\right)$, that the magnetic field strength $b_{i}$ at the inner edge of the disk is measured in units of $10 \mathrm{G}$ and that the disk accretion rate $\dot{m}_{a}$ is measured in units of $10^{-7} M_{\odot} \mathrm{yr}^{-1}$.

We emphasize that the energy density in the magnetic field of the disk never exceeds that of the thermal energy density. If we take the parameters of the boundary layer for disks calculated by Bertout, Basri, \& Bouvier (1988), for example, then a field that is in equipartition with the gas pressure in the boundary layer is of order $500 \mathrm{G}$. In this case our parameter $\epsilon_{i}$ still lies in the range $0.1-1.0$. We also note that for the PP solutions, $\epsilon_{i} \propto r_{i}^{1 / 2}$.

The innermost radius of the disk from which wind can be driven is taken to be $0.1 \mathrm{AU}$ since such stars may have strong surface magnetic fields that prevent a disk from reaching the stellar surface. T Tauri stars have typical radii of $R_{*} \simeq 4 R_{\odot}$, and strong stellar fields of perhaps $10^{3} \mathrm{G}$ can terminate the disk at an innermost radius (the magnetopause) of $R_{M} \simeq$ $5 R_{*} \equiv r_{i}$ (Bertout et al. 1988; Konigl 1991).

Magnetic flux surfaces in the outflow are labeled by the value of $a$ that they have. This value is most easily assigned at each radius $r_{0}$ where each flux surface cuts the accretion disk. A natural choice is the power-law distribution on the disk,

$$
a \propto \psi=\left(r_{0} / r_{e}\right)^{3 / 2 \alpha},
$$

where $\psi$ is the dimensionless poloidal flux. The choice (3.3) is quite general; the self-similar (BP) model is for $\alpha=2$ and by varying, $\alpha$, a wide variety of non-self-similar flows can be generated.

\subsection{Alfvén Surface}

The solution for the Alfvén surface is

$$
\frac{r_{\mathrm{A}}\left(r_{0}\right)}{r_{0}}=f_{\mathrm{A}}(\alpha) \frac{\lambda^{1 / 2}}{\epsilon} \psi^{2-\alpha} \propto r_{0}^{(3 / \alpha)-(3 / 2)},
$$

where $f_{\mathrm{A}}$ is a function of the index $\alpha$ and is of order unity. Notice that for $\alpha=2, r_{\mathrm{A}} / r_{0}=$ const, and therefore the flow is self-similar (BP). For more general flux distributions, however, the shape of the Alfven surface is not a cone.

A second important aspect of this solution is the scaling of the Alfven surface with the control parameters of the problem. From equations (3.2) and (3.4) we see that $r_{\mathrm{A}} / r_{0} \propto\left(B_{i}^{2} / \dot{M}_{a}\right)$. Thus, if the disk field weakens, the Alfven surface is strongly affected and moves closer to the axis of the outflow thereby reducing the lever arm of the flow. However, a decrease in the disk accretion rate $\dot{M}_{a}$ with time would lead to an increase in the lever arm (since the flow would have to carry off more disk angular momentum).

\subsection{Terminal Speed of the Flow}

The terminal speed along any field line in the flow is $2^{1 / 2} \Omega_{\mathrm{K}}(a) r_{\mathrm{A}}(a)$ which, knowing the Alfvén surface, gives

$$
v_{p, \infty}=2^{1 / 2} v_{\mathrm{K}, e} f_{\mathrm{A}} \frac{\lambda^{1 / 2}}{\epsilon} \psi^{2-(4 \alpha / 3)} \propto r_{0}^{(3 / \alpha)-2} .
$$

For self-similar flows $(\alpha=2)$, the terminal speed of the flow originating a position $r_{0}$ on the disk scales as the Keplerian speed, $v_{\infty} \propto v_{\mathrm{K}}\left(r_{0}\right)$ (as in BP). Disk winds have the property that a wide range of terminal speeds of material in the wind is achieved depending upon where in the disk corona the flow started. As an example, for $\alpha=3$ flows from Keplerian disks, material accelerated from $0.1 \mathrm{AU}$ on the disk achieves terminal speeds 10 times larger than gas that is accelerated from $1 \mathrm{AU}$.

We note that the terminal speed scales as

$$
v_{p, \infty} \propto B_{e}^{2} / \dot{M}_{a},
$$

so that a drop in the disk magnetic field with time implies a decrease in the disk wind speed. This is true because the Alfven surface has moved closer to the flow axis reducing the lever arm as discussed above. Conversely, a decrease in the accretion rate leads to a higher outflow velocity since the lever arm would then have increased.

\subsection{Mechanical Luminosity of the Wind}

The total mechanical luminosity of the flow is

$$
L_{w}=\frac{1}{2} \frac{G \dot{M}_{a} M_{*}}{r_{i}}\left[1-\left(\frac{r_{i}}{r_{0}}\right)\right] .
$$


This result shows if there is no dissipation of the gravitational binding energy within the disk, the flow can extract up to half the total gravitational binding energy that can be released in the accretion process in the form of mechanical energy of the wind. A hydromagnetic wind is an excellent extractor of gravitational binding energy released in the disk accretion flow. The result says that $L_{w} / L_{\text {bol }} \simeq$ const if the bolometric luminosity is largely due to accretion flow. Even if realistic dissipative effects are included, such as heating due to ambipolar diffusion of the disk field, the wind still extracts more than $90 \%$ of the gravitational binding energy (Konigl 1989).

The total mechanical luminosity of the winds with our choice of mass-loss rates and inner disk radii is then

$$
L_{w}=2.16 \times 10^{-1} \dot{m}_{a}\left(M_{*} / M_{\odot}\right) r_{i}^{-1} L_{\odot},
$$

where $r_{i} \simeq r_{M} \equiv\left(R_{M} / 0.1 \mathrm{AU}\right)$. Note however, that the observed optical luminosity of jets is

$$
L_{\text {optical }} \simeq 10^{-1} \text { to } 10^{2} L_{\odot}
$$

(Appenzeller \& Mundt 1989). Thus the observed optical properties of jets can be accounted for if about $10 \%$ of the mechanical jet power is converted to radiation through shocks (the exact fraction depends upon the accretion rate which is observationally not very well determined).

\subsection{Fixing the Free Parameters}

It is generally expected that outflows achieve a minimum energy configuration. This state implies that the toroidal field in the outflow scales as $B_{\phi} \propto r^{-1}$. There is correspondingly $a$ unique distribution of magnetic field across the accretion disk that can produce this minimal energy outflow, namely, $\alpha=3$. In this case, the magnetic field distribution across the accretion disk scales as $B_{0}\left(r_{0}\right) \propto r_{0}^{-3 / 2}$. This physical constraint is used to fix all the expressions for the terminal speed. Alfvén surface, etc., in the previous sections. It is important to realize that, unlike BP, this disk wind is non-self-similar.

The two free parameters of the theory are fixed by imposing the physical constraint that the disk wind be the driver of larger scale bipolar $\mathrm{CO}$ outflow. From this constraint, PP deduced the fiducial values

$$
\epsilon_{i}=0.24 ; \quad \lambda^{1 / 2}=3.17,
$$

where we adopt the value of $\epsilon_{i}$ derived from equation (3.2). However, the optical jet data (see $\S 3.8$ ) suggests that there is a range of values of $\lambda$, with the smallest value being about $\lambda^{1 / 2}=2.20$. Adopting this choice, our two disk wind parameters are fixed. The only free parameter left to adjust in this problem arises from the shocks, to which we turn later.

\subsection{Physical Quantities of the Flow}

The "lever" arm in centrifugally driven winds is the ratio of the Alfvén radius of the flow $r_{\mathrm{A}}\left(r_{0}\right)$ to the disk radius at which a particular field line threads the disk $\left(r_{0}\right)$, or

$$
\frac{r_{\mathrm{A}}\left(r_{0}\right)}{r_{0}}=3.05\left(\frac{\lambda^{1 / 2}}{9.17 \epsilon_{i}}\right)\left(\frac{r_{i}}{r_{0}}\right)^{1 / 2} .
$$

This value for the lever arm for flow from the inner part of the disk is consistent with the value derived by Kenyon \& Hartmann (1989). Flow originating from a disk radius of $r_{0}$ therefore achieves a terminal speed of

$$
v_{\infty}\left(r_{0}\right) \equiv v_{1}\left(r_{0}\right)=500\left(\frac{r_{i}}{r_{0}}\right)\left(\frac{\lambda^{1 / 2}}{9.17 \epsilon_{i}}\right) \mathrm{km} \mathrm{s}^{-1}
$$

along the field line. The preshock terminal speed drops to 25 $\mathrm{km} \mathrm{s}^{-1}$ for flow that originates from $2 \mathrm{AU}\left(=20 R_{M}\right)$ so that emission lines are likely only produced from flow that originated between 0.1 and $2.0 \mathrm{AU}$ on the disk.

The wind mass-loss rate $\dot{M}_{w}$ is related to the accretion rate in the disk (Pudritz 1985). These two rates are related by the square of the "lever arm" of the flow that for self-similar solutions gives

$$
\dot{M}_{w}\left(r_{0}\right)=2.71 \times 10^{-9}\left(9.17 \epsilon_{i} / \lambda^{1 / 2}\right)^{2} \dot{m}_{a}\left[\left(r_{0} / r_{i}\right)-1\right] M_{\odot} \mathrm{yr}^{-1}
$$

Given that the characteristic radius of the energetic outflow is (using the result in PP)

$$
r_{e}=\frac{r_{i}}{\epsilon_{i}^{2}} \simeq 1.74 \times 10^{13} \frac{b_{i}^{4}\left(r_{i} / 10^{12} \mathrm{~cm}\right)^{6}}{m \dot{m}_{a}^{2}} \mathrm{~cm},
$$

then the total mass-loss rate in the wind, integrating over the entire strong wind zone on the accretion disk, is

$$
\dot{M}_{w}\left(r_{e}\right)=4.45 \times 10^{-8} M_{\odot} \mathrm{yr}^{-1}
$$

for the stated accretion rate. This fiducial mass-loss rate agrees with estimates from the forbidden line profiles obtained by Edwards et al. (1987).

Once sees from equations (3.11) and (3.10) that the fiducial total mass-loss rate in the wind scales as

$$
\dot{M}_{w}\left(r_{e}\right) \propto \dot{M}_{a},
$$

so that a decrease in the disk accretion rate is directly related to a drop in the total mass-loss rate in the wind. It is interesting that the strength of the disk field is irrelevant for this scaling.

One finds that the density scales as $\rho \propto f(\psi) r^{-2}$ where $f$ is a function of the flux that is given in PP. Applying the results in PP we find that for $\alpha=3$, the neutral wind density in the regime beyond the Alfvén surface is given by

$$
\begin{aligned}
N_{1}\left(r_{0}\right)=2.4 \times & 10^{8} \frac{\dot{m}_{a}}{(\mu / 2.3)}\left(\frac{\epsilon_{i}}{0.24}\right)\left(\frac{9.17 \epsilon_{i}}{\lambda^{1 / 2}}\right)^{2} \\
& \times\left[\left(\frac{n}{1.80}\right)^{2} 0.54+0.34\right]^{-1}\left(\frac{r_{0}}{r}\right)^{2} \mathrm{~cm}^{-3},
\end{aligned}
$$

where $\mu$ is the reduced mass and where the FM number $n$ is scaled in terms of the critical value (1.80) for recollimating flow (see $\S 3.8$ ).

As will be shown in detail in $\S 5.2$, forbidden line emission implies wind densities not much larger than $N_{1} \simeq 10^{7} \mathrm{~cm}^{-3}$ in the centermost part of the shocked gas. From equation (3.12) we see that this is consistent if the flow diverges by an amount $\left(r_{0} / r\right) \simeq 0.20$ in going from the disk, and recollimating to the shock. This divergence is self-consistent if the size of the shocked gas region is of order the Alfvén radius in size. This is confirmed by calculating the size of the Alfven radius for the innermost part of the outflow, which is (from eq. [3.10]) $\left[r_{i} / r_{\mathrm{A}, i}\left(r_{i}\right)\right]=0.33$.

The wind density is sensitive to the combination of disk accretion rate and inner disk field strength,

$$
N_{1}\left(r_{0}\right) \propto \dot{M}_{a}^{4} / B_{i}^{6}
$$

A decrease in the fiducial disk field at constant accretion rate would lead to a much higher wind density. 


\subsection{Focal Points}

The general solutions of PP show that flows of high enough fast magnetosonic (FM) Mach number $n>n_{T}$ recollimate to the axis at a finite scale height above the accretion disk, while flows with $1 \leq n \leq n_{T}$ only achieve cylindrical flow. The critical FM number for recollimation is (for $\alpha=3$ )

$$
n_{T} \simeq\left[\left(2^{3 / 2} \lambda^{1 / 2}\right)-3\right]^{1 / 2} .
$$

For conditions applicable to our disk winds, we find that for flows characterized by our fiducial model parameters, $\epsilon_{i}=0.24$ and $\lambda^{1 / 2}=2.20$, recollimation occurs if the FM Mach number exceeds $\eta_{T} \simeq 1.80$.

The focal length of the flow can be found by noting that the flow radially converges at the Alfvén speed in the toroidal field $\left[v_{\mathrm{A}, \phi}=B_{\phi} /(4 \pi \rho)^{1 / 2}\right]$. Since $\rho \propto\left(r_{0} / r\right)^{-2}$ and the dominant toroidal field must scale as $B_{\phi} \propto r^{-1}$, the transverse Alfvén speed is faster for the inner parts of the flow as compared to the outer parts. Thus, the flow converges at the Alfvén speed in the transverse direction which scales with the terminal speed of the flow;

$$
\frac{v_{\mathrm{A}, \phi}\left(r_{0}\right)}{v_{\infty}\left(r_{0}\right)}=\mathrm{const}
$$

(using eq. [3.9]).

The focal length follows from the ratio of $v_{\infty} / v_{\mathrm{A}, \phi}$, which can be calculated from the results in PP. We find that the focal ratio does not depend upon the magnetic flux distributions across the central accretion disk and just depends upon the FM number,

$$
\frac{z_{\mathrm{foc}} / 2}{r_{\max }}=\frac{v_{\infty}}{v_{\mathrm{A}, \phi}}=\left[3\left(n_{1}^{2}+2\right)\right]^{1 / 2},
$$

where a fiducial radius of the flow, $r_{\max } \simeq r_{e} \simeq 2 \mathrm{AU}$. Combining this result with equation (3.14) therefore shows that (1) the FM number $n_{1}$ is a constant of the flow, and (2) the focal distance is constant as is the angle between the converging field lines and the flow axis $\theta_{f}$. For the fiducial parameter values discussed previously $\left(n_{1} \simeq 1.8\right)$, the focal distance is approximately $z_{\text {foc }}=7.9 r_{\max } \geq 16$ AU.

The field lines in the preshock gas converge toward the focal point and make an angle of

$$
\theta_{f}=\tan ^{-1}\left(2 r_{\max } / z_{\text {foc }}\right) \simeq 14.2
$$

with respect to the flow axis (we have used the fiducial parameters in evaluating eq. [3.16]).

Observational constraints on the distance between the two focal points (where the forbidden lines are formed) are that any such regions are separated by less than $\simeq 160 \mathrm{AU}$ (this corresponds to an angular separation of 1"0 between two sources placed at the distance of Taurus; $160 \mathrm{pc}$ ) which implies that $z_{\text {foc }} \leq 80 \mathrm{AU}$. It is interesting to note, however, that in many TTSs, the center of the emission region is offset from the central source by 0"1-0".5 (Solf 1989; Appenzeller \& Mundt 1989). From equation (3.15), this corresponds roughly to a range in FM number $m_{1}$ of $1.8-4.5$, or to a range of $\lambda^{1 / 2}$ between 2.2 and 8.1. More detailed results require line profile calculations.

\section{SHOCKS AT MAGNETIC FOCAL POINTS}

Recollimation shocks occur at a focal radius $r_{\text {foc }} \simeq r_{\mathrm{A}}$. Thus, the focal region is at a much smaller radius than the radius of the FM surface. The reason is that the agent that is responsible for the shock is the restoration of Keplerian rotation as the super-Alfvénic flow narrows. The shock occurs because the flow cannot narrow any further than $r_{\mathrm{foc}} \simeq r_{\mathrm{A}}$. We specifically prove this in Appendix A.

The toroidal field in the focal (and shock) region is in fact small compared to the poloidal component, $\left(B_{\phi} / B_{p}\right)_{\text {foc }} \ll 1$. While it is true that for flow radii larger than the FM surface $(n=1)$, the toroidal field is large (in the limit of large Alfvénic Mach number $m \gg 1$, then $B_{\phi} / B_{p} \simeq m / n \gg 1$ ) one knows that in general the toroidal field is small compared to the poloidal field component when one is in regions comparable to or smaller than the Alfven radius. This point is also proved in Appendix A.

Mathematically, the convergence of the flow leads to the appearance of nozzle points in the flow equations. If the preand postnozzle flow conditions cannot be matched, a shock must arise.

We present an illustration (from Ouyed 1992) of the acceleration and recollimation of a hydromagnetic disk wind with the consequent formation of a shock in Figure 4. The figure also includes the shock geometry as used in the following analysis.

\subsection{MHD Shock Jump Conditions}

Purely hydrodynamic models of the formation of forbidden line emission in TTSs require that the shock is highly oblique if most of the kinetic energy in the wind is not to be dumped into unacceptably high amounts of heat. Hartmann \& Raymond (1988) noted that if a stellar wind of $200 \mathrm{~km} \mathrm{~s}^{-1}$ impinges on the disk, then only a small part of its kinetic energy $(\simeq 40 \mathrm{~km}$ $\mathrm{s}^{-1}$ ) goes into the heating that produces the observed line

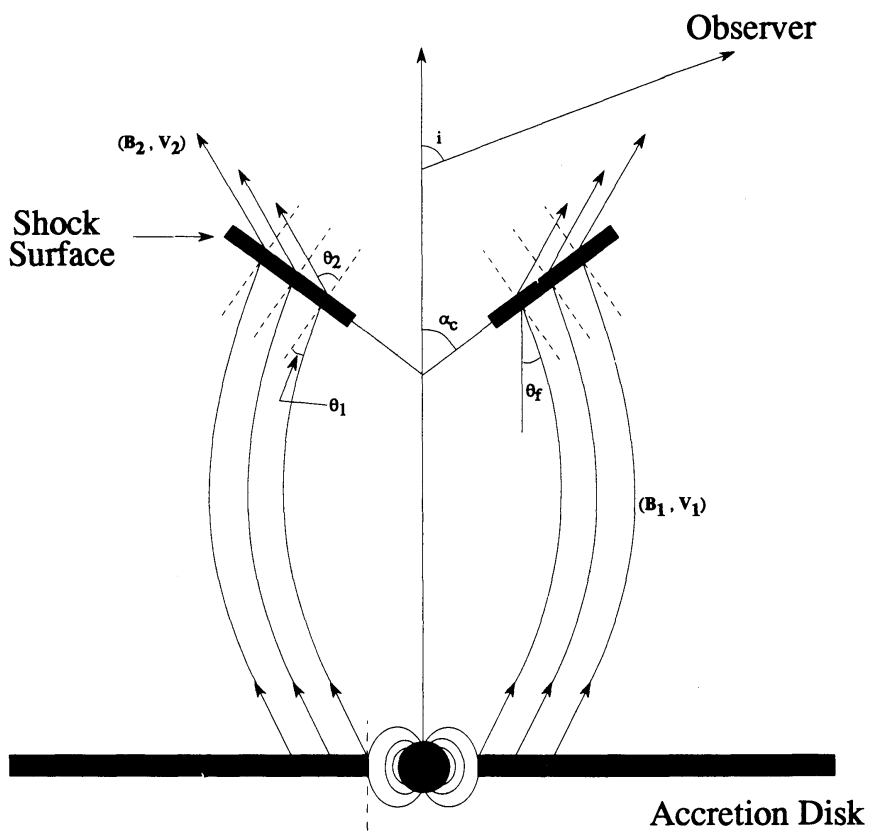

$0.1 \mathrm{AU}$

Fig. 4.- Illustration of the recollimation of a centrifugally driven, hydromagnetic disk wind, showing the shock geometry; reproduced from Ouyed (1992). The inner radius of the disk is at $0.1 \mathrm{AU}$ and the shock surface stands away from the disk at a height of $16 \mathrm{AU}$, for flows with FM Mach numbers of $n_{1}=1.8$. 
emission. Thus, any hydrodynamic theory must carefully arrange a shock that is a largely grazing blow. The important new feature about an MHD shock is that substantial flow energy goes into the amplification of the postshock magnetic field.

We model the MHD shocks in this paper as being adiabatic. We expect this to be a reasonable assumption because observations show that the outflow and postshock gas have low ionization (e.g., Natta et al. 1988). There are two channels for the preshock kinetic energy in adiabatic shocks, namely thermal and magnetic energy. If the gas stays neutral, one cannot soak up the shock energy in the ionization of gas and the resulting shock can be well described in the adiabatic approximation. We shall assume this for the rest of the paper.

We denote all pre- and postshock variables with the suffixes 1 and 2, respectively, and components perpendicular to and tangent with the shock front at each point by the suffixes $n$ and $t$, respectively. The mass flux as well as the magnetic flux being conserved through the shock implies that

$$
\begin{aligned}
\rho_{1} v_{n, 1} & =\rho_{2} v_{n, 2}, \\
B_{n, 1} & =B_{n, 2} \equiv B_{n} .
\end{aligned}
$$

Decomposing the equations of motion into components normal to and tangential to the shock,

$$
\begin{array}{r}
\rho v_{n}\left[v_{T}\right]-\frac{B_{n}}{4 \pi}\left[B_{T}\right]=0, \\
\left(\rho v_{n}\right)^{2}\left[\frac{1}{\rho}\right]+[p]+\frac{1}{8 \pi}\left[B_{T}^{2}\right]=0 .
\end{array}
$$

The requirement that the tangential component of the field be continuous through the shock (where $\boldsymbol{E}=-\boldsymbol{v} \times \boldsymbol{B} / \mathrm{c}$ ) gives another relation between tangential quantities,

$$
B_{n}\left[\boldsymbol{v}_{\boldsymbol{T}}\right]-\rho v_{n}\left(\frac{\boldsymbol{B}_{\boldsymbol{T}}}{\rho}\right)=0 .
$$

Finally, the energy equation for the gas can be reduced to an equation for the shock adiabatic for a magnetized fluid,

$$
\epsilon_{2}-\epsilon_{1}=\left(\frac{1}{2}\right)\left[\left(p_{2}+p_{1}\right)+\frac{\left(B_{T, 2}-B_{T, 1}\right)^{2}}{8 \pi}\right]\left(\frac{1}{\rho_{1}}-\frac{1}{\rho_{2}}\right) \text {. }
$$

This completes the set of MHD shock jump conditions required for our analysis.

\subsection{A Special Case: Switch-On Shocks}

In general, an MHD shock is oblique, that is, the preshock gas will make an angle $\theta_{1} \neq 0$ with respect to the shock normal. We expect, however, that the tangential component in the preshock field is small,

$$
B_{T, 1} \simeq 0, \quad B_{T} \equiv B_{T, 2} .
$$

This, as already discussed, is expected in the preshock gas of the focal region (by our analysis in Appendix A); the toroidal field (i.e., the tangential component) is small compared to the poloidal field in the preshock gas in the focal region. A much more detailed analysis including oblique shocks will be presented elsewhere (Ouyed \& Pudritz, 1993).

For our case, then, $\theta_{1}=0$, which is known in the literature as the so-called switch-on shock. This special case, as is well known, is especially simple since it is completely tractable ana- lytically (see, e.g., Landau \& Lifshitz 1960). We summarize the basic points below, leaving the details to Appendix B.

It is convenient to define a parameter which measures the energy in the preshock thermal gas pressure compared to magnetic pressure,

$$
\beta_{1} \equiv \frac{8 \pi p_{1}}{B_{1}^{2}}
$$

Writing $p_{1}=\rho_{1} c_{s, 1}^{2}$ and using the scaling for $\rho_{1}$ and $B_{1} \simeq$ $B_{\phi}$, one finds that $\beta_{1} \propto c_{s, 1}^{2} r_{0}^{2}$. In general, therefore, $\beta_{1}$ may vary from field line to field line.

For the special case that the sound speed in the wind scales as $c_{s}\left(r_{0}\right) \propto r_{0}^{-1}, \beta_{1}=$ const. This is a particularly simple scaling since it also implies that the wind everywhere has a constant sonic Mach number $v_{\infty} / c_{s}=$ const. In general, we expect that the parameter $\beta_{1}$ is small because it has a negligible value in the disk corona and is insignificant in driving the outflow (BP and PP). It is the remaining free parameter in our theory of forbidden line formation.

We show in Appendix B (eq. [B2]) that the density jump in the shock is $\rho_{2} / \rho_{1}=n_{1}^{2}$. Since $n_{1}$ is a constant throughout the preshock flow, we see that the density jump across the shock is constant over the whole shock surface. Thus variations in the shocked gas density can only arise because of spatial variations in the preshock wind density. The temperature jump $T_{2} / T_{1}$ is also a constant over the whole shock surface as long as the preshock $\beta_{1} \simeq$ const (see eqs. [B10] and [B11]).

The total postshock gas speed $v_{2}$ is exactly the Alfvén speed in the postshock fluid, $v_{2}=B_{2} /\left(4 \pi \rho_{2}\right)^{1 / 2}$ (eq. [B6]). The drop in velocity as one goes across the shock is found to be $v_{2} / v_{1}=$ $n_{1}^{-2} B_{2} / B_{1}$.

The magnetic field jump $B_{2} / B_{1}$ across the shock is a constant independent of the value of $\beta_{1}$. This implies that the postshock field lines all flare out with respect to the flow axis at the same angle. In the limit of cold MHD preshock flow that we are considering $\left(\beta_{1} \ll 1\right)$, the ratio $B_{2} / B_{1}$ achieves a maximum value at a value of the compression $\rho_{2} / \rho_{1}=$ $\gamma /(\gamma-1)$ for which

$$
\left(\frac{B_{2}}{B_{1}}\right)_{\max }=\left[\frac{\gamma}{(\gamma-1)}\right]^{1 / 2}=1.58 \quad\left(\gamma=\frac{5}{3}\right) .
$$

If $\theta_{2}$ is the angle of he postshock field with respect to the preshock field direction, then the maximum deflection angle of the postshock field lines from the shock normal direction is

$$
\theta_{2, \max }=\tan ^{-1}\left[\left(B_{T, 2} / B_{1}\right)_{\max }\right]=\tan ^{-1}\left[(\gamma-1)^{-1 / 2}\right] \text {. }
$$

For a $\gamma=5 / 3$ gas, this maximum angle is $\theta_{2} \leq 50^{\circ} 8$.

The density jump in the postshock gas is modest for an adiabatic strong shock because in order for $B_{2} / B_{1} \geq 1$, the density jump must be limited to

$$
1 \leq \frac{\rho_{2}}{\rho_{1}} \leq \frac{(\gamma+1)}{(\gamma-1)}
$$

This is the same limit as for purely hydrodynamic adiabatic shocks. For $a \gamma=5 / 3$ gas, the maximum compression is 4 .

The postshock temperature, pressure, speed, and magnetic field strength (derived analytically in Appendix B) specialized to a $\gamma=5 / 3$ gas are plotted as functions of $\rho_{2} / \rho_{1}=n_{1}^{2}$ for various values of $\beta_{1}$ in Figures $5 a-5 d$, respectively. The postshock density jump cannot be any larger than a factor of 4 for the strongest adiabatic shocks, so that the Alfvénic Mach 


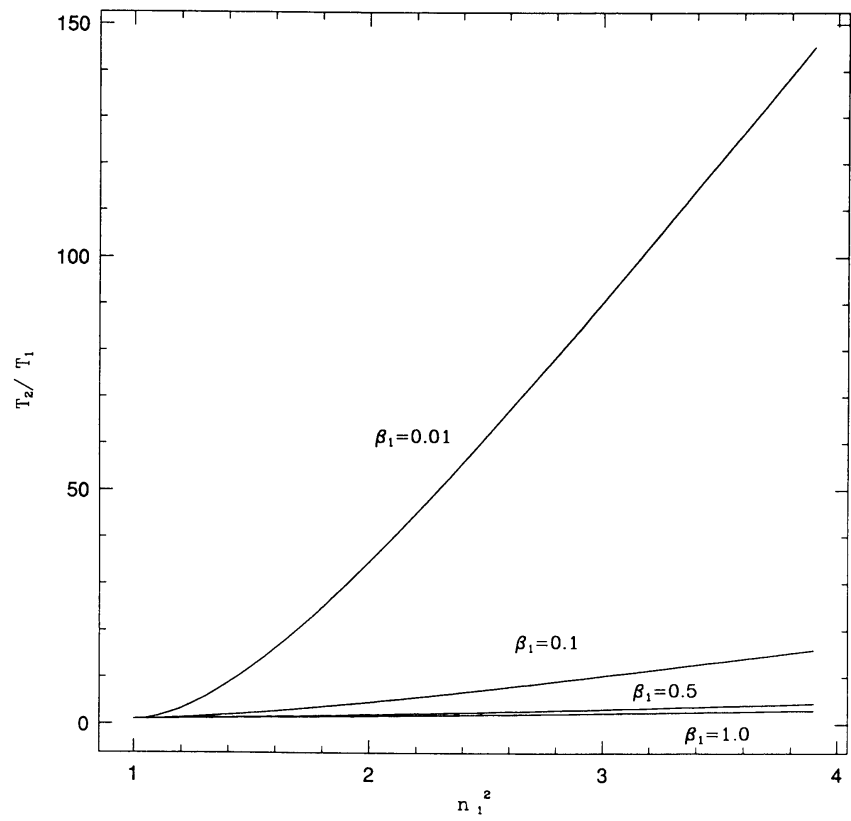

FIG. $5 a$

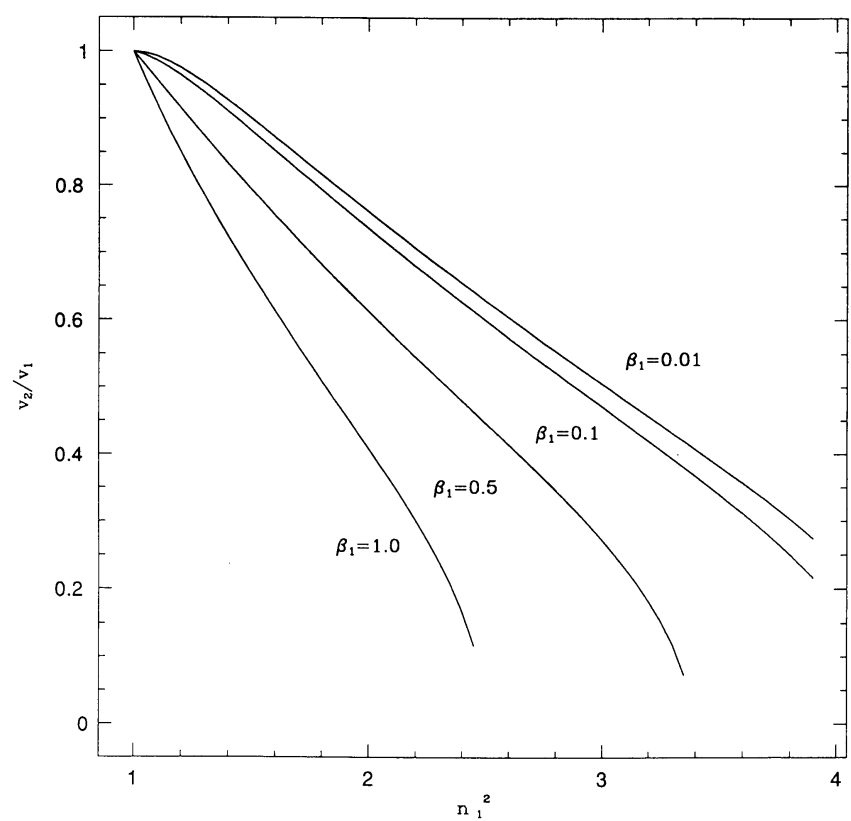

Fig. $5 c$

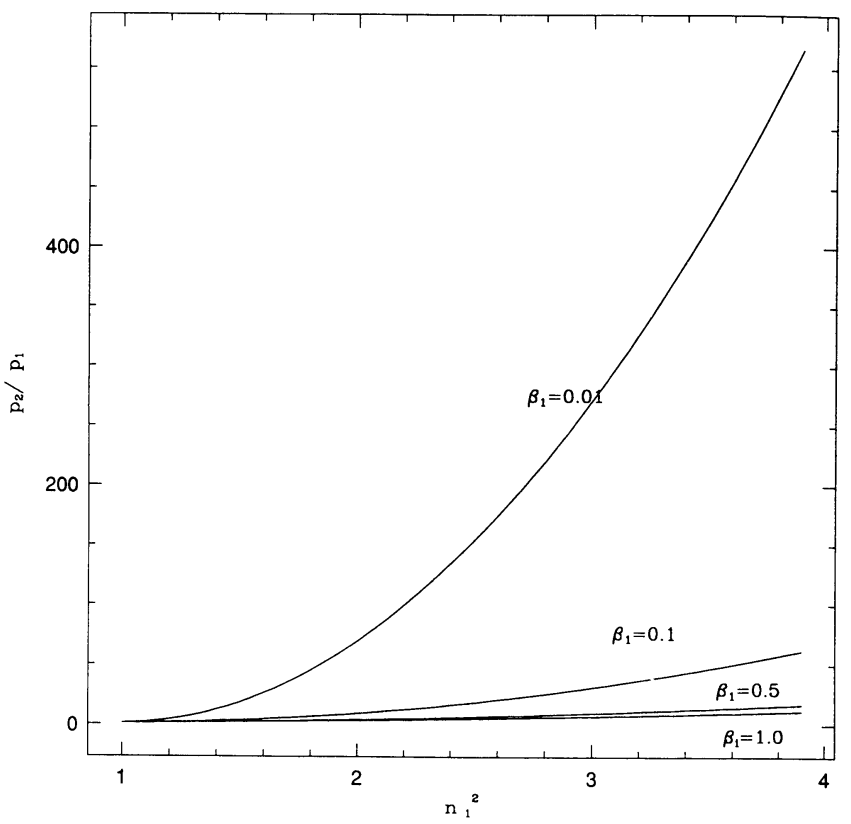

Fig. $5 b$

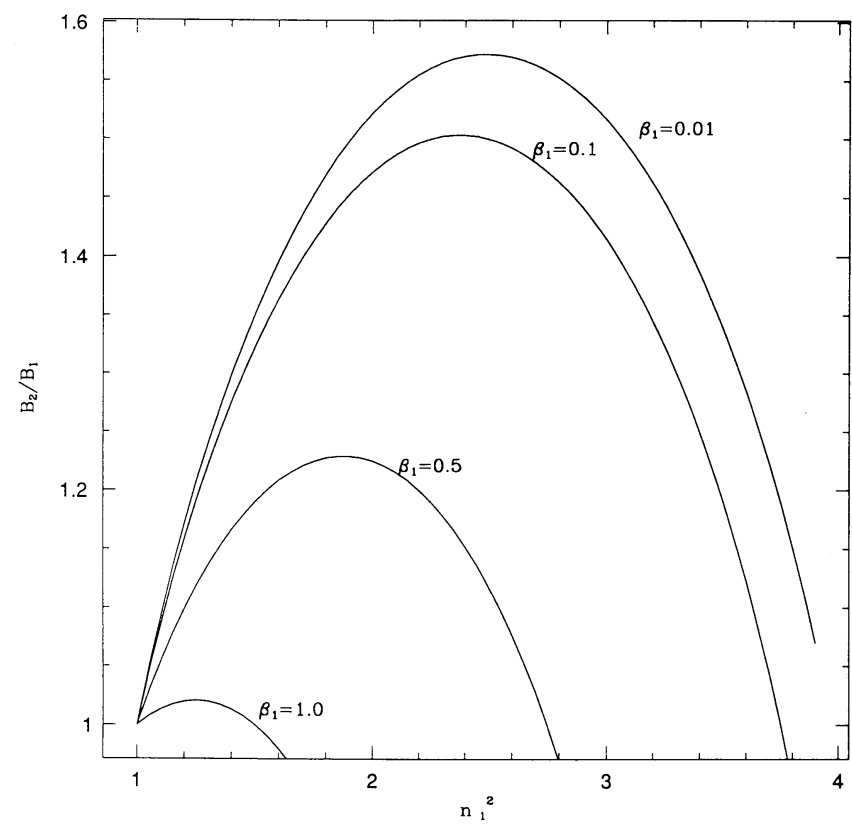

Fig. $5 d$

FIG. 5.- (a) Ratio of post- to pre-shock temperature as a function of the density jump $\rho_{2} / \rho_{1}=n_{1}^{2} ;(b)$ same as $(a)$ except for the pressure ratio $p_{2} / p_{1} ;(c)$ same as $(a)$ except for velocity ratio $v_{2} / v_{1} ;(d)$ same as $(a)$ except for magnetic field ratio $B_{2} / B_{1}$.

number is limited to $n_{1} \leq 2$. It should be remembered that the FM number $n_{1}$ is approximately $n_{1} \simeq n_{T}$.

Figure $5 a$ shows that the temperature jump for cold preshock flow is large for $\beta_{1} \simeq 0.01$, being of order 100 . Since the preshock gas is no more than several hundreds of degrees and excitation of the lines requires shocked gas to be at $\simeq 10^{4} \mathrm{~K}$ (see next section), we therefore confirm that cold preshock MHD flows imply the right temperature regime for forbidden line emission. The pressure jump across the shock (Fig. $5 b$ ) is a comparably large factor because the density contrast in the adiabatic shock is not larger than 4 (for an adiabatic index of $5 / 3)$.

We also plot the ratio $v_{2} / v_{1}$ in Figure $5 c$. From the curves we see that for $\beta_{1}=0.01$,

$$
v_{2} / v_{1}=0.5 \quad\left(n_{1}^{2}=3\right), \quad=0.3 \quad\left(n_{1}^{2}=4\right) .
$$

Since the preshock speeds are given by equation (3.10), we infer that for the above compressions the postshock speeds for the 
innermost streamlines of the flow are in the range $250-150 \mathrm{~km}$ $\mathrm{s}^{-1}$, as constrained by the observations.

Figure $5 d$ shows that a maximum field strength is produced in the shock depending on the compression ratio. For $n_{1}^{2} \simeq 2.5$ this achieves a maximum for $\beta_{1}=0.01$.

\subsection{General Case: Oblique MHD Shocks}

More extensive calculations of general oblique shocks reveal that in order to have Alfvén Mach numbers $n_{1} \geq 2$, the shock must be oblique. Ouyed \& Pudritz (1993) and Ouyed (1992) generally find that line profiles are best fitted with $\theta_{1} \leq 47^{\circ}$ for the case of $n_{1}=2$.

\section{EMISSION-LINE FORMATION IN SHOCKS}

We now demonstrate that the previously described disk wind and MHD shock parameters lead naturally to the formation of double-peaked optical forbidden lines. Specifically, we show that the range of densities and temperatures found in our shocked disk wind model accommodates the wide range in excitation conditions implied by the presence of [O I], [S II], and $[\mathrm{N}$ II $]$ emission.

\subsection{Temperature}

The forbidden lines detected in the TTS winds correspond to low-ionization species: [O I], [O II $],[\mathrm{S}$ II $],[\mathrm{N}$ II $]$ and [Fe II] (see, e.g., Appenzeller \& Mundt 1989). As the conditions for collisional excitation of the [O III] are similar to those for [O II], the lack of detection already indicates that the range of temperatures generated in the shock cannot be very large. We show that the shocks expected in recollimating flows are mild enough that these higher ionized species do not appear.

For the low densities $\left(N_{e} \leq 10^{8} \mathrm{~cm}^{-3}\right)$ and weak radiative fields involved, the ionization state of the gas can be described by what is usually called "coronal ionization equilibrium" (see, e.g., McCray 1987). In this state the elements are ionized only by thermal electrons. Thus, for optically thin gas, the steady state ionization balance condition gives rise to an expression for the number of $z$-stage ions. This can be cast into equations connecting adjacent ionization states

$$
N_{z+1} \alpha_{z+1, z}=N_{z} c_{z, z+1},
$$

where $N_{z+1}$ and $N_{z}$ represent the number density of ions in the ionization state $z+1$ and $z$, respectively, $\alpha_{z+1}$ is the recombination coefficient that includes both the radiative and the dielectronic recombination terms, and $c_{z, z+1}$ is the collisional ionization coefficient. The collisional recombination and radiative ionization rates are considered to be small in comparison with the foregoing rates. All the coefficients are functions of temperature alone. Therefore the ionization equilibrium constrains the temperature range for the shock.

We concentrate on the $\mathrm{O}^{+n}$ and $S^{+n}$ series because the strongest emission lines [O I] and [S II] are associated with them. The collisional ionization coefficients have been taken from Aller (1984) and the recombination from Aldrovandi \& Péquignot (1973). As is shown in Figure 6, the range of temperatures in which a considerable fraction of $[\mathrm{S}$ II] but not [O $\mathrm{III}]$ is produced is quite small. In addition, the strength of the [O $\mathrm{I}]$ lines implies that the temperature of the postshock gas cannot be very high. From Figure 6 we estimate that the postshock temperature of the gas is within the range,

$$
T_{2} \simeq 1.1-1.5 \times 10^{4} \mathrm{~K} .
$$

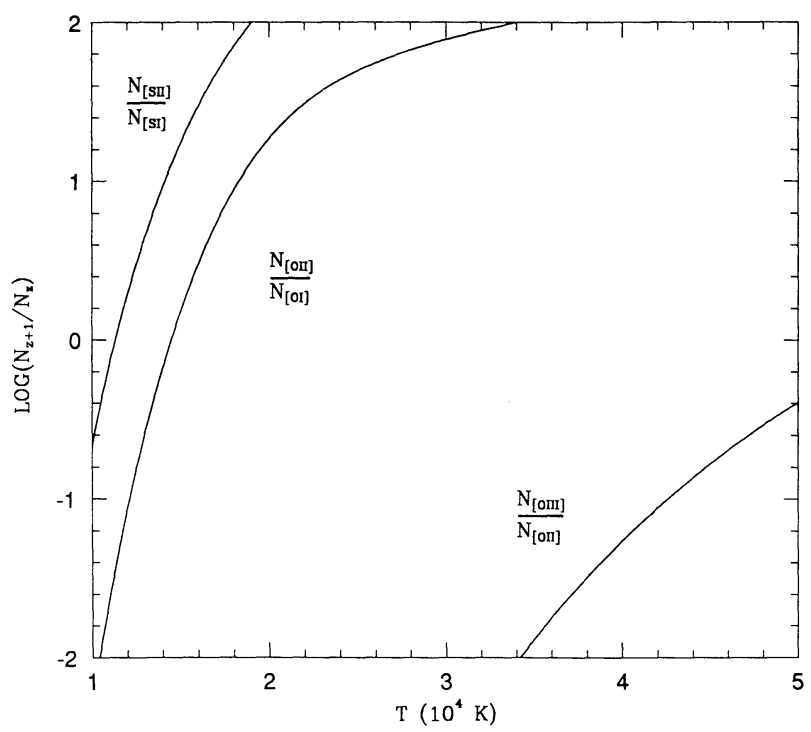

Fig. 6.- Ionization conditions in forbidden line emission regions

The detection of $[\mathrm{N} \mathrm{II}]$ in some sources, together with the very

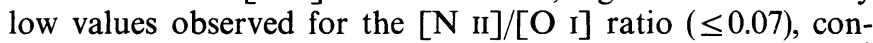
strains the temperature of the postshock gas to, $T_{2} \leq 1.2 \times 10^{4}$ $\mathrm{K}$. For this value, the ionization fraction in the wind is very small with an upper limit $f \leq 15 \%$ for the objects in which $[\mathrm{N}$ II $]$ is detected.

Some of the best evidence for the existence of neutral outflow is the work of Mitchell et al. (1988) who discovered high-speed $\left(\simeq 150 \mathrm{~km} \mathrm{~s}^{-1}\right)$ warm CO on scales less than $10^{16} \mathrm{~cm}$ from a massive young star. The authors suggest that the $\mathrm{CO}$ is heated by colliding with warm dust grains in the outflow.

Given that the appearance of singly ionized species such as [S II] is so sensitive to the temperature, why are the temperatures so "fine-tuned" to give lines of mild excitation, and not species such as [O III] in this model? The basic point is that when a flow acquires the critical value of $n_{1} \simeq n_{T}$ required for recollimation, it focuses and shocks. We might anticipate that $n_{1}$ will drop a little before hitting the shock, but it is clear that

$$
n_{1} \simeq n_{T} \simeq 1.8
$$

This is very modest value for the Mach number. It is the fact that recollimation occurs at such modest Mach numbers that ensures that the shock is not too strong, and that therefore high temperatures do not occur. The preshock Mach number is fixed at the specific value at which the flow starts to recollimate. Thus, the temperature of the shocked gas depends only upon the parameter $\beta_{1}$. The plots in Figure $5 a$ clearly show that for a given $n_{1}$, a temperature boost of about $10-100 \AA$ for $0.01 \leq \beta_{1} \leq 0.1$. If the preshock gas is too cold (i.e., $\beta_{1}$ is smaller), the calculation shows that the amount of energy dumped into the postshock gas is too high, and temperatures that would allow [O III] production could appear. Finally, we recall that our shock theory has $T_{2} / T_{1}=$ const (for constant $\left.\beta_{1}\right)$.

The observations can therefore be explained by saying that the wind originates from a disk corona with $\beta_{1} \simeq 0.01-0.1$. This range of values is in line with theoretical ideas about magnetized disk coronae (e.g., BP). 


\subsection{Densities}

The electronic densities inferred from the [S II] lines are $\simeq 10^{4} \mathrm{~cm}^{-3}$. This value is very close to the critical electronic density $\left(N_{\mathrm{cr}}=1.3 \times 10^{4} \mathrm{~cm}^{-3}\right)$ at which the forbidden lines become rapidly weaker because collisional de-excitation increases. However, as pointed out by Edwards (1987), the

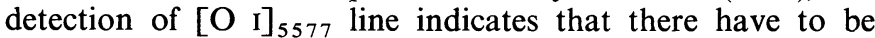
regions with electronic densities as high as $N_{e} \simeq 10^{7} \mathrm{~cm}^{-3}$ in the wind. Therefore, there is a broad range of electronic densities $\simeq 10^{4}-10^{7} \mathrm{~cm}^{-3}$, in the shock region.

The excitation conditions of the TTSs can be reproduced over a wide range of particle densities, depending only on the amount of free energy available that can be dumped into the gas. For shock temperatures of the order of $10^{4} \mathrm{~K}$ the densities can vary between $10^{4}$ and $10^{6} \mathrm{~cm}^{-3}$ reproducing the observed lines ratios (Hartmann \& Raymond 1988). The ionization fraction varies from $15 \%$ to $0.1 \%$ in this range. At larger densities the $[\mathrm{O} \mathrm{I}]_{5577}$ becomes very intense while the rest of the lines cannot be observed any longer. Note that at low densities the $6300 \AA$ line is much more intense than the $5577 \AA$. However at high electronic densities, $N_{e} \simeq 10^{6-7} \mathrm{~cm}^{-3}$, the lower levels are thermalized and only the $5577 \AA$ is observed. This implies that the electronic density of the wind varies over three orders of magnitude.

The size of the region that radiates the forbidden lines can be estimated from the emission measure of the optically thin, radiating gas (see, e.g., Edwards et al. 1987). Noting that our wind density is much larger than the one pictured by these authors, we find applying their formula to our higher density region that

$$
r_{\text {forbid }}=0.66 \mathrm{AU}\left[\frac{\left(N_{\mathrm{cr}} / N_{2}\right)}{10^{-3}}\right]^{-2 / 3}
$$

for gas at a density of $10^{7} \mathrm{~cm}^{-3}$ and at a critical ionization level of $X_{\mathrm{cr}} \simeq 10^{-3}$. Of course, any emission from a more extended jet would be in addition to that from the focal region.

\subsection{Shock Geometry and Double-Peaked Line Profiles}

A striking feature about the forbidden lines is that they are generally double-peaked, with peaks separated by $200 \mathrm{~km} \mathrm{~s}^{-1}$ (Edwards 1987, 1989). One of the peaks is typically found at zero velocity, while the other is almost always blueshifted. Since it is impossible for this feature of the forbidden lines to arise as a consequence of self-absorption, a possible explanation is that the lines are formed on a conical surface with a wide opening angle $\alpha_{c}$ as measured from the flow axis (Edwards et al. 1987). The idea here is that for sufficiently wide opening angles, a typical observer will see gas moving at different projected speeds from the two sides (foreground and background) of this surface. These authors find that for angles smaller than $30^{\circ}$, the separation between the peaks is much smaller than is observed. The best fits appear for $70 \geq \alpha_{c} \geq 40^{\circ}$ $\left(\alpha_{c}=90^{\circ}\right.$ is a hemispheric wind). However, geometric effects alone are not sufficient to explain the double-peaked profiles since recent work has shown that the slower velocity component is produced in lower density gas than the higher velocity component (Mundt et al. 1990). Our model readily accounts for this since the lower velocity material in the pre- (and post-) shock gas is at lower density. It is also farther from the jet axis.

We have already found from our HD shock theory that the postshock field lines are deflected making large angles with respect to the preshock field line direction. From equation (4.9) this angle is

$$
\theta_{2} \leq 51^{\circ} \text {. }
$$

Thus the opening angle that the outflowing gas in the postshock region with respect to the flow axis is

$$
\alpha_{c}=\theta_{2}-\theta_{f} \simeq 40^{\circ} .
$$

Thus, it appears that the MHD shock theory naturally explains the origin of double-peaked line profiles as the expanding postshock gas.

\section{DISCUSSION AND CONCLUSIONS \\ 6.1. Correlations with the IR Excess}

One of the most thoroughly worked-out ideas for the origin of the IR excess is by the reprocessing of UV and optical photons by a surrounding dusty disk or envelope. The source of the UV photons is usually taken to be a hot boundary layer.

The infrared excess generated through accretion has been studied in detail (e.g., Adams, Lada, \& Shu 1987; Bertout et al. 1988). Normally it is assumed that disks are dominated by viscous torques and are heated by the dissipation of gravitational binding energy released during the accretion process. The sources of the excess are the radiation from the disk with typical temperatures in the range of $1300-300 \mathrm{~K}(R \leq 1 \mathrm{AU})$, and the reprocessing of the UV radiation released in the boundary later near the star. In this way it is possible to reproduce $K-L$ colors in the range 0.4-0.9 (Bertout et al. 1988). In our diagram, this range of infrared colors characterizes our type II stars. From this point of view, the correlation shown in Figure 1 represents the increase of the wind strength with the accretion rate. This we expect to occur in accretion-driven disk winds.

The reprocessing of UV photons from the boundary layer cannot explain the excess of sources with $K-L>1.0$, however (e.g., Bertout et al. 1988). These sources are located in region A of our diagram and are characterized as having jets and/or strong forbidden line emission. One needs to look for another source of energy to explain this extreme excess.

We propose that the excess in energy arises from the energy released in such shocks. We recall from section $\S 3.2$ that the winds can be $90 \%$ efficient in extracting gravitational binding energy released during disk accretion. Thus estimates for the accretion rate through YSO disks meausure only that part of the accretion responsible for dissipation in the disk, which may be as little as $10 \%$ of the total rate. The apparently unexplained IR excess then naturally arises from energy in the disk wind that is dissipated in shocks and produces optical photons.

The IR excess may arise from the reprocessing of optical photons released at the shock and intercepted by the dusty accretion disk. This is plausible since the recollimation shock stands about $20 \mathrm{AU}$ off the disk surface, while the disk itself may be at least $100 \mathrm{AU}$ in extent. Disks must have enough optical depth to hide the redshifted forbidden line emission. Reprocessing can also occur by the dense, dusty material in the swept-up gas shell around the bipolar flow. Finally we note that the high density of the disk wind allows the formation of small dust grains in the outflow that may also contribute to the opacity in the shock region (Gomez de Castro 1993).

While it is beyond the purpose of this paper to calculate in detail, the energetics of the IR excess can certainly be explained on the basis of outflow energy released in the shocks. This naturally accounts for the correlation of equivalent line width with infrared excess. 


\subsection{Evolution of Sources}

How must sources evolve if we are to account qualitatively for the trend in Figure 1? The observations suggest that protostellar systems start with higher accretion rates that are gradually reduced with time. The hydromagnetic disk wind theory we have employed predicts that the total mass-loss rate in the wind scales with the disk accretion rate, $\dot{M}_{w}\left(r_{e}\right) \propto \dot{M}_{a}$. Thus, if protostellar disks evolve toward lower accretion rates, the wind mass-loss rate must be reduced with time. This fits in well with our current understanding of mass-loss trends in TTSs.

If the accretion rate falls with time, then we can use the scalings of disk wind theory given in $\S 3$ for the terminal speed $v_{\infty}$ and wind density $N_{1}\left(r_{0}\right)$ to predict evolution of the flows. We recall from equation (3.8) that $v_{\infty} \propto \epsilon^{-1} \propto B_{e}^{2} / \dot{M}_{a}$. Thus jet velocity is also sensitive to the magnetic field in the disk. The field may also decay with time due to the process of ambipolar diffusion. Using a convenient expression for the ambipolar diffusion time given by McKee et al. (1993),

$$
t_{\mathrm{amb}}=0.74 \times 10^{5}\left(K_{i-1} / 1\right)\left(10^{8} / n_{\mathrm{H}}\right)^{1 / 2} \mathrm{yr},
$$

we find that the diffusion time is of order $2.2 \times 10^{4} \mathrm{yr}$ for the expected condition of disks. Thus, the reduction of surface density and disk magnetic field can be expected to occur on time scales of order $10^{4}-10^{5} \mathrm{yr}$.

Thus, it is likely that both the disk accretion rate and the fiducial field strength decrease with time. Unfortunately, there is still no reliable observational or theoretical result by which $\dot{M}_{a}$ can be computed as a function of time. If we speculate that $B_{i}^{2} / \dot{M}_{a}$ increases with time, which is to say that the mass accretion rate falls more quickly with time than the disk field, then $v_{\infty}$ will increase with time. This would imply that the magnetic focal point remains a fixed feature of all the sources in our Figure 1 . At the same time, the wind density $N_{1} \propto \dot{M}_{a}^{4} / B_{i}^{6}$ will likely strongly decrease with time. The optical forbidden line emission would then gradually disappear because the gas becomes both hotter and less dense during the evolution of the disk to smaller field and accretion rate. Finally, equation (3.11) informs us that the jet radius would strongly increase as time goes on, becoming perhaps at least $20 \mathrm{AU}$ in width. We emphasize that without further constraints, the opposite case to the above might also be true; the terminal speed could decrease with time, which would have the effect of discouraging shocks. Clearly observational constraints are required on the disk accretion rate as a function of time before we can make more precise statements.

\subsection{Conclusions}

We have shown that the data argue against stellar hydromagnetic winds as the drivers of the underlying outflows. The reason is that the optical emission lines and stellar rotation speeds appear to be completely uncorrelated with one another. Since in any model the emission lines are produced as a consequence of outflow, and since rotation is an important ingredient of a centrifugal wind, stars seem to be an untenable source for the winds. Keplerian disks on the other hand, make excellent centrifuges, and we have shown that such winds may constitute the simplest mechanism that is consistent with all of the ever growing number of observational facts.

We have employed a comprehensive theory of hydromagnetic winds from Keplerian accretion disks (PP) that shows that such flows naturally recollimate and form MHD shocks at focal points. We have shown that the properties of the wind, as well as those of the MHD shock, readily explain a wide variety of the known facts about forbidden line emission in young stellar objects. The underlying wind theory depends upon only two parameters. These are chosen in such a way so so as to explain the observed thrust seen in larger scale $\mathrm{CO}$ outflows. We have shown that the conditions in such a disk wind are then ideal for the formation of forbidden lines at the focal points.

The theoretical model predicts that recollimation of the flow occurs when it reaches a very modest magnetic (FM) Mach number of $n_{1} \simeq 1.8$. Flows that don't achieve this value only collimate into cylindrical flows and do not shock. The highly collimated disk outflow achieves very high densities $\simeq 10^{7}$ $\mathrm{cm}^{-3}$ in the inner regions of the flow $(0.1 \mathrm{AU})$ and drops to $\simeq 10^{4} \mathrm{~cm}^{-3}$ in the outer parts of the flow $(2 \mathrm{AU})$. In adiabatic MHD shocks, the density increase across the shock is at most a factor of 4 , so the range in densities probed by the forbidden lines reflects the large range in density that occurs in the underlying disk wind. We anticipate that the [S II] lines which are excited at modest densities $\left(\simeq 10^{4} \mathrm{~cm}^{-3}\right)$ are produced in the outer parts of the shock $(1 \mathrm{AU})$ while the $\left[\mathrm{O}_{\mathrm{I}}\right]_{5577}$ lines are produced, under the aforementioned conditions, in the innermost portions $(0.3 \mathrm{AU})$.

The focal points themselves are not much larger than $\simeq 0.5$ $\mathrm{AU}$ in radius and stand above and below the disk at about a distance of $\geq 16 \mathrm{AU}$. Typical shock temperature of $10^{4} \mathrm{~K}$ are readily achieved, and we used a simple, adiabatic MHD shock theory to analytically derive most properties. Higher excitation species such as [O III] are not produced if the preshock flow is sufficiently magnetized; $0.01 \leq \beta_{1} \leq 0.1$.

The gas remains largely neutral through the shock, and the electron fraction is $X_{e} \simeq 10^{-1}$. The primary advantage of MHD shocks over hydrodynamic ones is that much of the flow energy goes into the formation of a strong, postshock field. Thus, the shocked gas does not have to be as emissive as in the purely hydrodynamic theory, even for shocks that are only slightly oblique. In this sense MHD shocks are much more robust and do not require fine tuning to reproduce the data.

We propose that fully developed optical jets originate at the focal points as well. Jets arise in those sources whose outflows could repeatedly recollimate and shock after the first focal point has been passed. Evolution of jets and FLERs depends both upon the evolution of the disk magnetic field, and the accretion flow through the disk. However, there is too little quantitative observational information available at this time to pin down the exact sense of the evolution.

The high efficiency of hydromagnetic disk winds in extracting gravitational binding energy implies that estimates of mass accretion rates in disks based on strictly viscous accretion models may underestimate the mass accretion rate in protostellar systems by factors of 5 or larger.

Finally, we suggest that the infrared excess of deeply embedded objects arises from the conversion of some of the mechanical energy in flows into shock heating. Reprocessing of the optical and UV photons produced in this manner, by dust in the dense accretion disk and/or swept-up shell the flow, would convert this flux into the observed IR excess.

We have benefitted from stimulating conversations with Sylvie Cabrit, Lee Hartman, Suzanne Edwards, Scott Kenyon, Reinhard Mundt, Rachid Ouyed, Guy Pelletier, and Alejandro Raga. We thank an anonymous referee for useful comments. 
R. E. P. acknowledges the receipt of a Reinhardt Fellowship from the Canadian Institute for Theoretical Astrophysics (CITA) that allowed this work to be completed. A. I. G. wishes to thank the Spanish Ministry of Education and NSERC for the receipt of International Postdoctoral Fellowships. This work was funded by a grant from NSERC of Canada.

\section{APPENDIX A}

\section{SHOCKS AT FOCAL POINTS}

We first determine the rotation frequency $\Omega$ of material at the radius of the focal point. To do this, we employ the solution for the toroidal velocity (again from ideal MHD), written as

$$
\Omega=\Omega_{*}(1-g),
$$

where the function $g$ is to be evaluated in the super-Alfvénic limit $m^{2} \gg 1$,

$$
g \simeq\left(r^{2}-r_{\mathrm{A}}^{2}\right) / r^{2}
$$

(these results corresponds to PP, eqs. [2.6] and [2.8], respectively). Equations (A1) and (A2) show that super-Alfvénic flow cannot focus to radii less than the Alfvén radius, since $\Omega \leq \Omega_{*}$ implies that $r \geq r_{\mathrm{A}}$. Thus, along each flow line, the radial position of the shock at the focal point is expected to be $r_{\text {foc }} \simeq r_{\mathrm{A}}(1+\epsilon)$ where $\epsilon \ll 1$.

We now evaluate the toroidal field of the preshock disk wind in the focal region. We first note that one of the basic consequences of the induction equation is Ferraro's law,

$$
B_{\phi} v_{p}=\left(\Omega-\Omega_{*}\right) r B_{p},
$$

which is reiterated in PP (eq. [2.4]). The relative strength of the toroidal to poloidal field components is then just

$$
B_{\phi} / B_{p}=\left(\Omega-\Omega_{*}\right) r / v_{p} .
$$

Substituting the expression for the angular velocity of the gas into that of the toroidal field, one then finds

$$
B_{\phi} / B_{p} \simeq\left[\Omega_{*} r_{\mathrm{A}} / v_{p}\left(r_{\mathrm{A}}\right)\right] 2 \epsilon
$$

at the focal radius. Noting from the Bernoulli theorem however that in this region $v_{p}=v_{\infty} \simeq 2^{1 / 2} \Omega_{*} r_{\mathrm{A}}$, we obtain

$$
B_{\phi} / B_{p} \simeq 2^{1 / 2} \epsilon \ll 1
$$

on each field line in the focal region.

\section{APPENDIX B}

\section{SWITCH-ON MHD SHOCKS}

The condition that there is a negligible tangential component of the preshock field allows us to eliminate the tangential velocity jump between equations (4.4) and (4.5) to find the relation

$$
\left(\rho v_{n}\right)^{2}=\rho_{2} \frac{B_{n}^{2}}{4 \pi}
$$

These are two important consequences of this result. Using equation (4.1) we find from equation (B1) that the density contrast across the shock is

$$
\frac{\rho_{1}}{\rho_{2}}=\frac{B_{n}^{2}}{4 \pi \rho_{1}} \frac{1}{v_{n, 1}^{2}} \simeq \frac{1}{n_{1}^{2}} .
$$

Another application of equation (4.1) to equation (B1) reveals that the normal component of the postshock gas moves off at the postshock Alfvén velocity,

$$
v_{n, 2}=\frac{B_{n}}{\left(4 \pi \rho_{2}\right)^{1 / 2}} .
$$

From the continuity equation and (B2), we then also have that

$$
\frac{v_{n, 1}}{v_{n, 2}}=n_{1}^{2} .
$$


We see that for large preshock magnetic Mach numbers $n_{1}$, the normal component of the flow is greatly reduced. However, the flow in going through the shock acquires a large tangential velocity component. This arises, as we shall see, because a large postshock tangential field develops. The postshock tangential speed is determined using equation (4.3),

$$
v_{T, 2}=\frac{B_{T}}{\left(4 \pi \rho_{2}\right)^{1 / 2}},
$$

where we have used equation (B2) as well. Thus, the postshock gas expands at the Alfvén speed in the tangential direction. The total speed of the postshock gas is therefore

$$
v_{2}=\left(v_{n, 2}^{2}+v_{T, 2}^{2}\right)^{1 / 2}=\frac{B_{2}}{\left(4 \pi \rho_{2}\right)^{1 / 2}},
$$

where we use equations (B4) and (B5), and where $B_{2}$ is the total postshock magnetic field strength.

We calculate the ratio of the post to preshock gas speed using equation (B6) to find

$$
\frac{v_{2}}{v_{1}}=\frac{1}{n_{1}^{2}} \frac{B_{2}}{B_{1}}
$$

where we remind the reader that $v_{1} \simeq v_{n, 1}$. Since the postshock gas must in general move more slowly than the preshock gas, this result shows that the field can be very strongly amplified in the shock, that is $B_{2} \leq n_{1}^{2} B_{1}$. The energy source for this is of course the kinetic energy of the preshock material.

The pressure jump across the shock is of direct observational significance since from the optical forbidden lines, one has some estimate for $\mathrm{N}_{2} \mathrm{~T}_{2}$. In order to find it in terms of the density jump (thereby finding the Hugoniot relation for magnetized gas), we must solve for the jump in the magnetic field strength across the shock. To do this, we use equation (4.4) and equation (B1) and find that

$$
\frac{B_{2}^{2}}{B_{1}^{2}}=\left(2 n_{1}^{2}-1\right)-\beta_{1}\left(\frac{p_{2}}{p_{1}}-1\right),
$$

where the ratio of thermal to magnetic energy in the preshock flow is

$$
\beta_{1} \equiv \frac{8 \pi p_{1}}{B_{1}^{2}} \ll 1 .
$$

The preshock plasma $\beta$ is small since our flow is a very cold MHD disk wind in which pressure plays an insignificant role in driving the gas. The value of the postshock tangential field is readily obtained from equation (B8) and $B_{T, 2}^{2}=B_{2}^{2}-B_{1}^{2}$.

We can now solve for the pressure jump across the shock. As is standard for hydrodynamic shock theory, one starts by writing down the energy equation for the gas and in so doing find, the shock adiabatic of the magnetized gas. From the internal energy equation (Landau \& Lifshitz), equation (4.6) in the text, one wants to solve for the magnetic Hugoniot adiabatic. As usual, one assumes the form for the internal energy

$$
\epsilon=\frac{p}{(\gamma-1) \rho}
$$

where $\gamma$ is the adiabatic index.

We can use equation (B8) to eliminate the magnetic field jump in equations (4.6) and (B9) to eliminate the pressure. After some algebra one finds the result that the pressure ratio across the shock is

$$
\frac{p_{2}}{p_{1}}=1+\left(n_{1}^{2}-1\right) \gamma+\frac{(\gamma-1)\left(n_{1}^{2}-1\right)^{2}}{\beta_{1}} .
$$

The results shows that the temperature jump across the shock is then

$$
\frac{T_{2}}{T_{1}}=\frac{p_{2}}{\rho_{2}} \frac{\rho_{1}}{p_{1}}=\frac{1}{n_{1}^{2}} \frac{p_{2}}{p_{1}} .
$$

We next calculate the amplification of the field in going through the shock. Substituting equation (B11) into equation (B8) one obtains

$$
\frac{B_{2}^{2}}{B_{1}^{2}}=-(\gamma-1) n_{1}^{4}+\gamma\left(2-\beta_{1}\right) n_{1}^{2}-\left(1-\beta_{1}\right) \gamma .
$$

Finally, we can calculate the postshock deflection $\theta_{2}$ of the magnetic field lines from the expression $\theta_{2}=\tan ^{-1}\left[B_{T, 2} / B_{1}\right]$. This may be calculated from equation (B12). 


\section{REFERENCES}

Achterberg, A., Blandford, R. D., \& Goldreich, P. 1983, Nature, 304, 607 Adams, F. C., Lada, C. J., \& Shu, F. H. 1987, ApJ, 321, 788

II Aldrovandi, S. M. V. \& Péquignot, D. 1973, A\&A, 25, 137

II Aller, L. H. 1984, in Physics of Thermal Gaseous Nebulae (Dordrecht: Reidel), 324

Appenzeller, I., \& Mundt, R. 1989, ARA\&A, 3, 56

Bertout, C., Basri, G., \& Bouvier, J. 1988, ApJ, 330, 350

Blandford, R. D., \& Payne, D. R. 1982, MNRAS, 199, 883 (BP)

Cabrit, S., Edwards, S., Strom, S. E., \& Strom, K. M. 1990, ApJ, 354, 687

Calvet, N., Hartmann, L., \& Kenyon, S. J. 1993, ApJ, 402, 623

Cohen, M., Emerson, J. P., \& Beichman, C. A. 1989, ApJ, 339, 455

Cohen, M., \& Kuhi, L. V. 1979, ApJS, 41, 743

Edwards, S., Cabrit, S., Strom, S. E., Heyer, I., Strom, K. M., \& Anderson, E. 1987, ApJ, 321, 473

Elias, J. H. 1978, ApJ, 224, 857

Gomez de Castro, A. I. 1993, A\&A, submitted

Hartigan, P., Hartmann, L., Kenyon, S., Strom, S. E., \& Skrutskie, M. F. 1990 ApJ, 354, L 25

Hartmann, L., Hewett, R., Stahler, S., \& Mathieu, R. D. 1986, ApJ, 309, 275

Hartmann, L., \& Raymond, J. C. 1988, ApJ, 337, 903

Hartmann, L. \& Stauffer, J. R. 1989, AJ, 97, 873

Herbig, G. H., Vrba, F. J., \& Rydgren, A. E. 1986, AJ, 91, 575

Heyvaerts, J., \& Norman, C. A. 1989, ApJ, 347, 1055

Hollenbach, D., \& McKee, C. F. 1989, ApJ, 342, 306

Jankovics, S., Appenzeller, I., \& Krautter, J. 1983, PASP, 95, 883

Kenyon, S., \& Hartmann, L. 1989, ApJ, 342, 1134

Konigl, A. 1989, ApJ, 342, 208 1991, ApJ, 370, L39

Lada, C. J. 1991, in NATO ASI, The Physics of Star Formation and Early Stellar Evolution, ed. C. J. Lada \& N. Kylafis (Dordrecht: Kluwer), 329

Landau, L. D., \& Lifshitz, E. M. 1960, Electrodynamics of Continuous Media (Toronto: Pergamon), chap. 54

Mathis, J. S., Rumpl, W., \& Nordsieck, K. H. 1977, ApJ, 217, 425
McCray, R. 1987, in Spectroscopy of Astrophysical Plasmas, ed. A. Dalgarno \& D. Layzer (New York: Cambridge Univ.), 255

McKee, C. F., Hollenbach, D. J., Seab, C. G., \& Tielens, A. G. G. M. 1987, ApJ 318,674

McKee, C. F., Zweibel, E. G., Heiles, C., \& Goodman, A. A. 1993, in Protostars and Planets III, ed. E. H. Levy, J. I. Lunine, \& M. Mathews (Tucson: Univ. Arizona Press), in press

Mitchell, G. F., Allen, M., Beer, R., Dekany, R., Huntress, W., \& Maillard, J.-P. 1988, ApJ, 327, L17

Mundt, R., Ray, T. P., Bührke, T., Raga, A. C., \& Solf, J. 1990, A\&A, 232, 37

Natta, A., Giovanardi, C., Palla, F., \& Evans, N. J., II, 1988, ApJ, 327, 817

Ouyed, R. 1992, M.Sc.thesis, McMaster Univ.

Ouyed, R., \& Pudritz, R. E. 1993, ApJ, submitted

Parker, N. D., Padman, R., \& Scott, P. F. 1991, MNRAS, 252, 442

Pelletier, G., \& Pudritz, R. E. 1992, ApJ, 394, 117 (PP)

Pudritz, R. E. 1985, ApJ, 293, 216

Pudritz, R. E., \& Norman, C. A. 1983, ApJ, 274, 677

Pudritz, R. E., Pelletier, G., \& Gomez de Castro, A. I. 1991, in NATO ASI, Physics of Star Formation and Early Stellar Evolution, ed. C. J. Lada \& N. Kyfalis (Dordrecht: Kluwer), 539

Rydgren, A. E., Strom, S. E., \& Strom, K. M. 1976, ApJS, 30, 307

Sakurai, T. 1985, A\&A, 152, 121

Shull, J. M., \& Draine, B. T. 1987, in Interstellar Processes, ed. D. J. Hollenbach \& H. A. Thronson, Jr. (Dordrecht: Reidel), 283

Solf, J. 1989, in ESO Workshop on Low-Mass Star Formation and Pre-MainSequence Objects, ed. B. Reipurth (Garching: ESO)

Skrutskie, M. F., Dutkevitch, D., Strom, S. E., Edwards, S., Strom, K. M., \& Shure, M. A. 1990, AJ, 99, 1187

Strom, K. M., Strom, S. E., Kenyon, S. J., \& Hartmann, L. 1988, AJ, 95, 534

Strom, K. M., Wilkin, F. P., Strom, S. E., \& Seaman, R. L. 1990, preprint

Walter, F. M., Brown, A., Mathieu, R. D., Myers, P. C., \& Vrba, F. J. 1988, AJ, 96, 297 